\title{
Long-Range Ordered Thin Films of Block Copolymers Prepared by Zone-Casting and Their Thermal Conversion into Ordered Nanostructured Carbon
}

\author{
Chuanbing Tang ${ }^{1}$, Adam Tracz ${ }^{2}$, Michal Kruk ${ }^{1}$, Rui Zhang ${ }^{1}$ \\ Detlef-M. Smilgies ${ }^{3}$, Krzysztof Matyjaszewski ${ }^{1}$, Tomasz Kowalewski ${ }^{1}{ }^{*}$ \\ ${ }^{1}$ Department of Chemistry, Carnegie Mellon University, 4400 Fifth \\ Avenue, Pittsburgh, Pennsylvania 15213. \\ ${ }^{2}$ Centre of Molecular and Macromolecular Studies, Polish Academy of \\ Sciences, 90-363 Lodz, Sienkiewicza 112, Poland. \\ ${ }^{3}$ CHESS, Cornell University, Ithaca, NY 14853
}

\section{Synthesis}

A diblock copolymer of $n$-butyl acrylate (BA) and PAN was prepared by chainextension from poly( $n$-butyl acrylate) macroinitiator to polyacrylonitrile using nitroxidemediated polymerizations as described earlier. ${ }^{1}$ The block copolymer had a number molecular weight of $37,500 \mathrm{~g} / \mathrm{mol}$ (with the structural formula $(\mathrm{BA})_{240}(\mathrm{AN})_{124}$ ) and polydispersity index of 1.22 (from gel permeation chromatography (GPC)). Generally block copolymers within this composition range tend to form bulk cylindrical morphologies rather than lamellar structures observed here by GISAXS in the samples prepared by zone-casting. ${ }^{2}$ In order to eliminate the possibility that this apparent discrepancy was caused by the erroneous composition characterization, the relative molar ratio of PAN and PBA segments was repeatedly determined with the aid of ${ }^{1} \mathrm{H}$ NMR and elemental analysis, which produced reproducible, consistent results. The compositions

reported herein represent the average value from ${ }^{1} \mathrm{H}$ NMR and elemental analysis. The determination of the overall absolute composition was based on the accurate measurement of molecular weight of the first block (PBA) obtained from GPC. ${ }^{3,4}$

\section{Sample Preparation}

Thin and ultra-thin copolymer films were prepared by drop-casting or zone-casting from $2 \mathrm{mg} / \mathrm{mL}$ solutions in $\mathrm{N}, \mathrm{N}$-dimethylformamide (DMF) onto silicon wafers covered with the layer of native oxide. Thermal stabilization and carbonization were carried out 
by heating the copolymer films to $280^{\circ} \mathrm{C}$ under air, followed by heating to $800^{\circ} \mathrm{C}$ under nitrogen. Zone casting was performed using a home-built apparatus, equipped with two computer controlled linear stages and independently-controlled solution and substrate heaters.

\section{Characterization}

Tapping Mode Atomic Force Microscopy TMAFM studies were carried out with the aid of a Nanoscope III-M system (Digital Instruments, Santa Barbara, CA), equipped with a J-type vertical engage scanner. The AFM observations were performed at room temperature in air using silicon cantilevers with nominal spring constant of $50 \mathrm{~N} / \mathrm{m}$ and nominal resonance frequency of $300 \mathrm{kHz}$ (standard silicon TESP probes). Typical value AFM detector signal corresponding to r.m.s. cantilever oscillation amplitude was equal to 1000 3000 mV and the images were acquired at $3 \mathrm{~Hz}$ scan frequency in $3 \times 3$ or $5 \times 5$ $\mu \mathrm{m}^{2}$ scan areas. PAN and PBA domains were particularly well contrasted in phase images, which provide 2-D maps of cantilever phase shift with respect to the drive. In imaging materials containing soft, mechanically lossy regions, contrast in phase images is dominated by energy dissipation in tip-sample interactions. ${ }^{5}$ In the current study, more lossy PBA phase exhibited stronger phase shifts, manifested as darker regions in phase images. Elongated, rigid PAN domains appeared as brighter regions in phase images and as protrusions surrounded by more compliant PBA phase in height images. Thin films prepared by simple drop-casting, exhibited characteristic striped meandering surface topography, consistent with lamellar morphology revealed by grazing incidence small angle x-ray scattering (GISAXS). The average domain spacing determined from radiallyaveraged 2-D Fourier transforms of AFM images and from GISAXS patterns was equal, respectively to 44 and $39 \mathrm{~nm}$ (the feature in GISAXS pattern was very broad and illdefined, c.f. Figure S3). The discrepancy between the spacings determined by the different techniques was most likely caused by the considerable curvature and branching of lamellae visible in AFM images. Although the stripes in AFM images exhibited a high degree of local parallelization, neither AFM images nor GISAXS patterns of films prepared by drop-casting showed any evidence of long range lamellar alignment. 
Film Thickness Measurements Films prepared by zone-casting exhibited periodic thickness fluctuations manifested as the presence of taller $\sim 100 \mu \mathrm{m}$ wide flat regions (stripes) spaced by approximately $100 \mu \mathrm{m}$. Such fluctuations are not uncommon in zone casting, and can be usually eliminated by careful matching of the rates of solvent supply, evaporation and substrate withdrawal. All observations reported herein were performed within the taller stripes. The film thickness in these regions was determined from AFM images taken in the vicinity of scratches made intentionally to expose the underlying silicon surface. Typically, for the samples zone-cast from $2 \mathrm{mg} / \mathrm{mL}$ copolymer solution, the film thickness was equal to about $130 \pm 30 \mathrm{~nm}$. After $800{ }^{\circ} \mathrm{C}$ pyrolysis, the film thickness was reduced to approximately $70 \pm 20 \mathrm{~nm}$.

Transmission Electron Microscopy (TEM) Carbonized films were lifted from the silicon substrate by immersion for a few hours in $25 \% \mathrm{HF}$, followed by floating onto the surface of ultrapure water from which they were transferred onto copper grids. The samples were examined using a Hitachi H-7100 electron microscope operating at $70 \mathrm{KV}$.

Raman Scattering The spectra were collected on a Jobin Yvon T64000 Raman system (ISA, Edison, NJ) with microprobe sampling optics. The excitation was at $514.5 \mathrm{~nm}(\mathrm{Ar}+$ laser, Model 95, Lexel Laser, Fremont, CA). The laser power was set to $33 \mathrm{~mW}$ and was focused on the sample to a spot diameter of approximately $3 \mu \mathrm{m}$. The back-scattered radiation entered the triple monochromator, which was operated in triple subtractive mode. The entrance slits to the monochromator and the spectrograph were set to $100 \mu \mathrm{m}$. A liquid nitrogen cooled CCD was used as the detector. Silicon sharp peak at $520 \mathrm{~cm}^{-1}$ was used as a frequency calibration standard. The total collection time per sample was approximately 300-500 seconds. Raman scattering spectra of carbon films prepared by pyrolysis of zone-cast copolymer films exhibited typical partially graphitic carbon characteristics, with D-band corresponding to disordered structures centered at $\sim 1350 \mathrm{~cm}^{-}$ ${ }^{1}$ and graphitic G-band centered at $\sim 1590 \mathrm{~cm}^{-1}$. ${ }^{6}$ Progressive partial graphitization of material with the increase of pyrolysis temperature was evidenced by the accompanying increase of the intensity of the G-band relative to the D-band.

Fourier Transform Infrared Spectroscopy The progress of PAN carbonization was also monitored with an FTIR-NIR Spectrometer (Mattson ATI Affinity 60AR). The FTIR spectra of block copolymer precursors exhibited a characteristic strong absorption 
band at $2850 \sim 2960 \mathrm{~cm}^{-1}$ corresponding to $\mathrm{CH}$ stretching vibration mode of PBA and PAN, a strong absorption at about $1735 \mathrm{~cm}^{-1}$ corresponding to $\mathrm{C}=\mathrm{O}$ stretching vibration mode of PBA, and a sharp absorption peak at approximately $2240 \mathrm{~cm}^{-1}$ corresponding to $\mathrm{C} \equiv \mathrm{N}$ groups of PAN. After thermal stabilization at $280^{\circ} \mathrm{C}$, the spectra showed substantial decrease of $\mathrm{CH}$ and $\mathrm{C}=\mathrm{O}$ absorption. The spectra of samples pyrolyzed to $600{ }^{\circ} \mathrm{C}$ and $800{ }^{\circ} \mathrm{C}$ exhibited the presence of broad and strong absorption feature between 1600-1300 $\mathrm{cm}^{-1}$, indicating the formation of $\mathrm{C}=\mathrm{C}$ and $\mathrm{C}=\mathrm{N}$ bonds derived from the cyclization and cross-linking reactions involving $\mathrm{C} \equiv \mathrm{N}$ groups. The observed red shift of $\mathrm{C}=\mathrm{C}$ and $\mathrm{C}=\mathrm{N}$ absorptions upon increase of pyrolysis temperature $\left(800{ }^{\circ} \mathrm{C}\right)$ is consistent with the increasing extent of conjugation. Meanwhile, the intensity of absorption band due to $\mathrm{C} \equiv \mathrm{N}$ groups diminished with the increase of pyrolysis temperature, to finally disappear following the treatment at $800{ }^{\circ} \mathrm{C} .{ }^{7}$

Grazing Incidence Small Angle X-ray Scattering (GISAXS) GISAXS images ${ }^{8}$ were taken at CHESS D station (Cornell University). A wide bandpass (1.7\%) double-bounce multilayer monochromator supplied an intense beam of $8 \mathrm{keV}$ photons which impinged onto the sample surface under various incident angles ranging from below the critical angle of the film and above the critical angle of the substrate. The sample was mounted on a sample goniometer, in order to control the incident angle and the sample azimuth. An accurate calibration of the incident angle was performed in-situ by measuring the $\mathrm{x}$ ray reflectivity from the sample using an ion chamber. GISAXS scattering intensities were recorded with an area detector (Medoptics) with a resolution of $50 \mu \mathrm{m}$ per pixel and a total area of about $50 \mathrm{~mm}$ by $50 \mathrm{~mm}$ at a distance of $1207 \mathrm{~mm}$ from the sample. The intense scattering in the incident plane was blocked with a $1.5 \mathrm{~mm}$ tantalum rod. Exposures times under these conditions ranged from $1 \mathrm{sec}$ to $30 \mathrm{sec}$ depending on contrast and sample quality. Data reported herein were obtained for the angle of incidence equal to $0.35^{\circ}$.

Electrical Conductivity Electrical conductivity measurements were performed using a four point probe method with Ti electrodes deposited on the films in ultra-high vacuum using electron-beam evaporation. 
Movie Tapping mode AFM phase images of PBA- $b$-PAN block copolymers prepared by zone-casting; Left image: scanned perpendicular to the casting direction; right image: scanned parallel to the casting direction. Insets: 2-D Fourier transform magnitude.

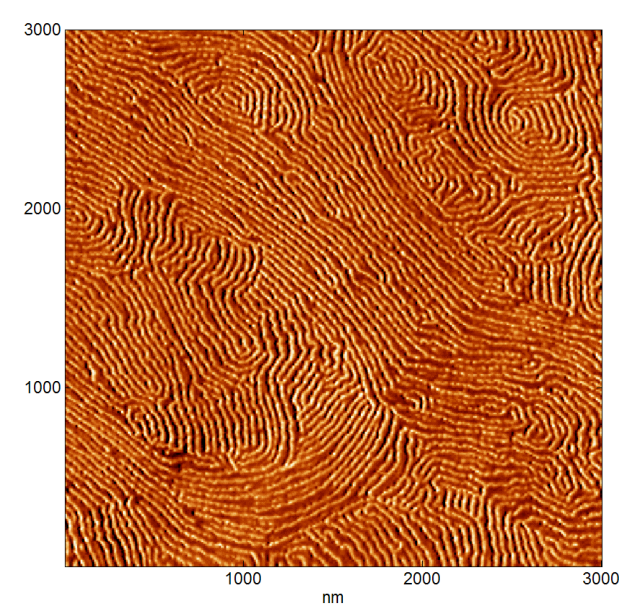

S1 Tapping mode AFM phase image of $(\mathrm{BA})_{240}(\mathrm{AN})_{124}$ prepared by drop-casting.

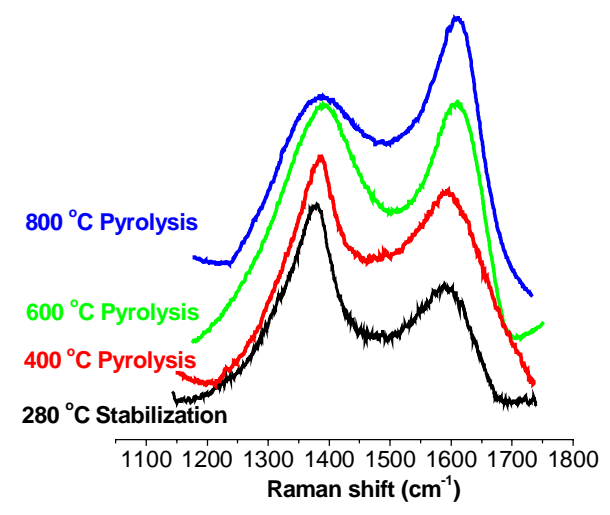

S2 Raman scattering spectra of thin carbon films prepared from zone-cast (BA) $240(\mathrm{AN})_{124}$ block copolymers acquired at different stages of thermal treatment (intensity: a. u.) 


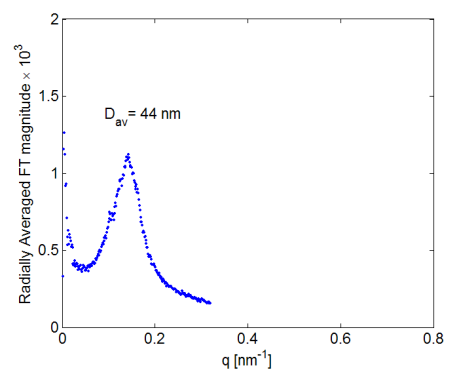

a)

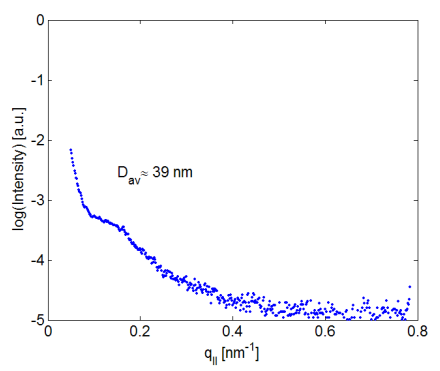

d)

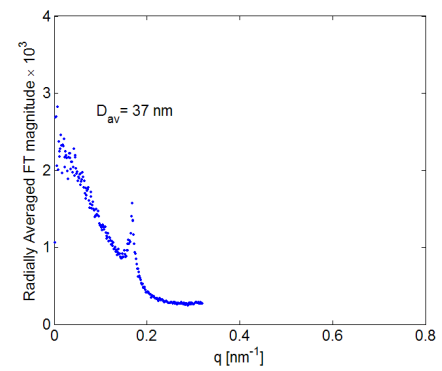

b)

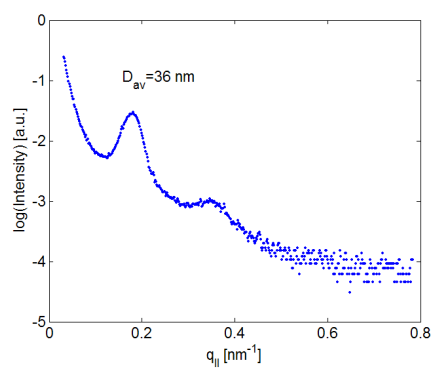

e)

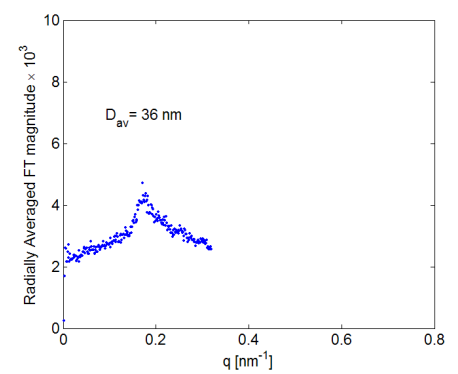

c)

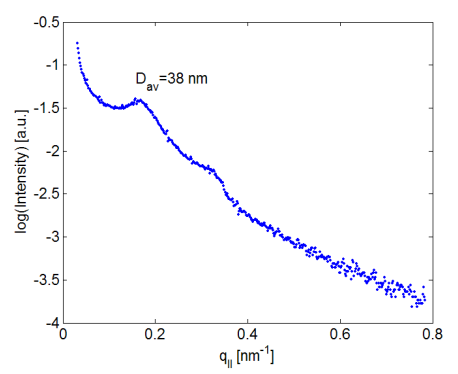

f)

S3 Radially averaged magnitudes of 2-D Fourier transform of AFM phase images and GISAXS scattering profiles $\left(\mathrm{q}_{\mathrm{z}}=0.32\right)$ for thin films of $(\mathrm{BA})_{240}(\mathrm{AN})_{124}$ block copolymers prepared by zone-casting and drop-casting and for carbon films derived from zone-cast copolymers. Top row - radially averaged magnitudes of 2-D Fourier transform of AFM phase images: a) drop-cast copolymer film; b) zone-cast copolymer film; c) carbon film prepared from zone-cast copolymer film. Bottom row - GISAXS profiles: d) drop-cast copolymer film; e) zone-cast copolymer film; f) carbon film prepared from zone-cast copolymer film 

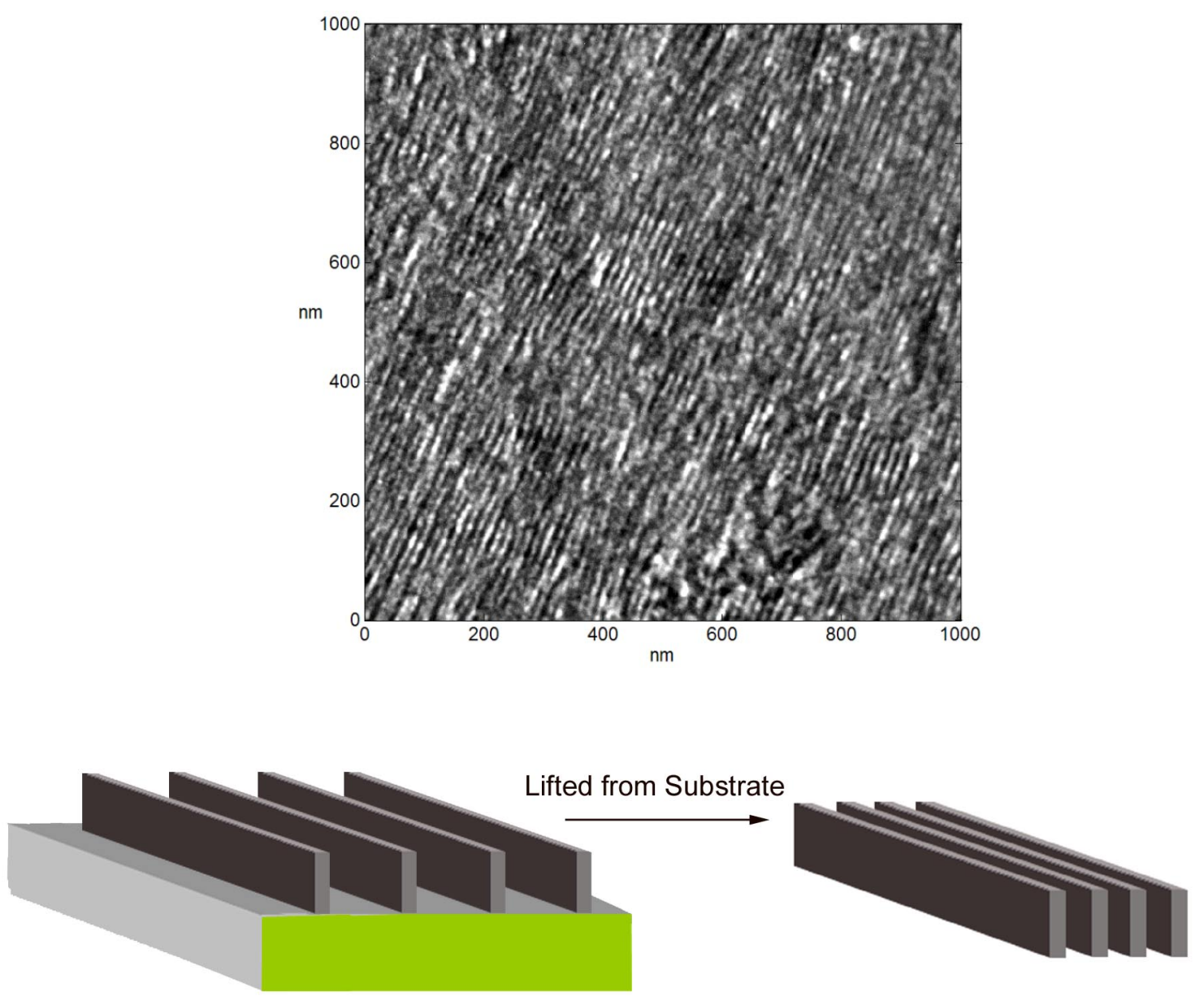

S4 TEM image of thin carbon film pyrolyzed at $800{ }^{\circ} \mathrm{C}$ from zone-cast PBA- $b$-PAN block copolymer thin film and schematic description of lamellar collapse upon lifting from the substrate. 


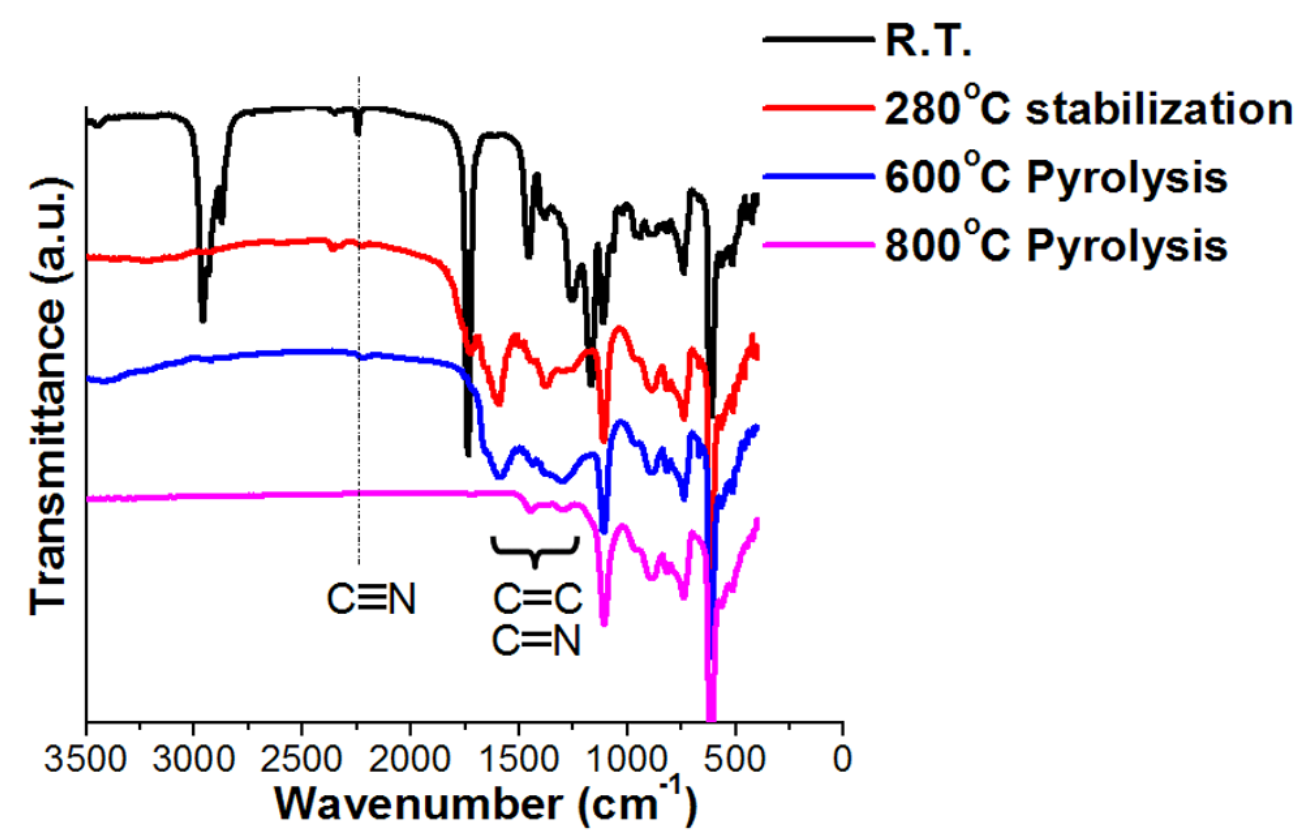

S5 FTIR spectra of thin films of zone-cast $(\mathrm{BA})_{240}(\mathrm{AN})_{124}$ block copolymers and resulting carbon acquired at different stages of thermal treatment on a silicon wafer.

\section{References}

(1) Tang, C.; Kowalewski, T.; Matyjaszewski, K. Macromolecules 2003, 36, 1465 1473

(2) Tang, C.; Smilgies, D-M.; Matyjaszewski, K. ; Kowalewski, T. Unpublished data

(3) Wang, J. S.; Matyjaszewski, K. J. Am. Chem. Soc. 1995, 117, 5614-5615

(4) Matyaszewski, K.; Nakagawa, Y.; Jasieczek, C. B. Macromolecules 1998, 31, 1535-1541

(5) Wu, W.; Matyjaszewski, K.; Kowalewski T. Langmuir 2005, 21, 1143-1148

(6) Tuinstra, F., Koenig, J.L. J. Chem. Phys. 1970, 53, 1126-1130

(7) Tang, C.; Qi, K.; Wooley, K.; Matyjaszewski, K.; Kowalewski, T. Angew. Chem. Int. Ed. 2004, 43, 2783-2787

(8) Smilgies, D.-M.; Busch, P.; Posselt, D.; Papadakis, C.M.; Synchrotron Radiation News 2002, 15, 35-41 